\title{
Vitamina D e prevenzione cardiovascolare
}

\author{
Luca Di Lullo ${ }^{1}$, Rodolfo Rivera ${ }^{2}$, Fulvio Floccari ${ }^{3}$, Vincenzo Barbera ${ }^{1}$, Antonio Bellasi ${ }^{4}$, Antonio De Pascalis ${ }^{5}$, Domenico Russo ${ }^{6}$, \\ Luigi Russo ${ }^{6}$, Francesca Santoboni ${ }^{1}$, Claudio Ronco ${ }^{7}$
}

${ }^{1}$ U.O.C. Nefrologia e Dialisi, Ospedale Parodi-Delfino, Colleferro (RM)

${ }^{2}$ Divisione di Nefrologia, Ospedale S. Gerardo, Monza

${ }^{3}$ U.O.C. Nefrologia e Dialisi, Ospedale S. Paolo, Civitavecchia (RM)

${ }^{4}$ U.O.C. Nefrologia e Dialisi, ASST Lariana, Como

${ }^{5}$ U.O.C. Nefrologia e Dialisi, Ospedale V. Fazzi, Lecce

${ }^{6}$ Cattedra di Nefrologia, Università degli Studi Federico II, Napoli

${ }^{7}$ International Renal Research Institute, Ospedale S. Bortolo, Vicenza

\begin{abstract}
Vitamin D and cardiovascular prevention
Vitamin $D$ represents the precursor of calcitriol, crucial for bone and mineral metabolism. The high prevalence of vitamin D deficiency and the detection of vitamin D receptor (VDR) both in the heart and vessels have focused the interest on potential cardiovascular effects of vitamin D.

Although several experimental data showed the protective role of vitamin D, on the other hand vitamin D "overdose" can contribute to heart and vascular calcification.

Meta-analyses of randomized-controlled trials (RCTs) underlined the association of vitamin D deficiency and increased cardiovascular morbidity and mortality but no significant improvements on cardiovascular outcomes are provided by the supplementation of vitamin $D$.

The following review provides an update on pathophysiology of vitamin $D$ and vitamin $D$ receptor (VDR) discussing on the association with cardiovascular risk factors and disease and on the role of vitamin $D$ supplementation in preventing cardiovascular disease in chronic kidney disease (CKD) patients.
\end{abstract}

Keywords: Chronic kidney disease (CKD), Observational studies, Randomized-controlled trials (RCTs), Vitamin D, Vitamin D receptor (VDR)

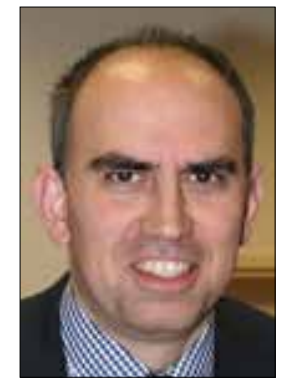

Luca Di Lullo

\section{Introduzione}

Il ruolo cruciale della vitamina $D$ nel metabolismo osseo e minerale è ben documentato sin dagli albori della medicina moderna e non solo (1-5). II passo successivo, e probabilmente ancora più determinante, è stato quello relativo all' identificazione di recettori per la vitamina D (VDR) a livello di diversi organi e tessuti, tra i quali il cuore e i vasi arteriosi.

Accepted: June 18, 2016

Published online: July 11, 2016

Indirizzo per la corrispondenza:

Dr. Luca Di Lullo

U.O.C. Nefrologia e Dialisi

Ospedale L. Parodi-Delfino

Piazza A. Moro 1

00034 Colleferro (RM)

dilulloluca69@gmail.com

Lo scopo della presente rassegna è quello di mettere in evidenza i rapporti esistenti tra livelli di vitamina $D$, patologia cardiovascolare e relativi fattori di rischio specifici attraverso l'analisi della più recente letteratura scientifica (metanalisi, studi epidemiologici e trial clinici randomizzati).

Ovviamente, la rassegna metterà in luce gli aspetti fondamentali del metabolismo della vitamina $D$ e quelli della malattia cardiovascolare, per poi evidenziare i rapporti tra attivazione dei VDR e i fattori di rischio cardiovascolare.

\section{Il metabolismo della vitamina D}

Dal punto di vista storico, la prima descrizione relativa alla vitamina $D$ va attribuita a McCollum, il quale la individuò quale fattore in grado di correggere la sintomatologia del rachitismo, caratterizzato da anomalie nella crescita ossea con relative deformità scheletriche (1).

Allo stato attuale, quella che McCollum chiamò vitamina $D$ in realtà è un precursore (quindi un pro-ormone) dell'ormone steroideo 1.25 diidrossi-vitamina $\mathrm{D}\left[1.25(\mathrm{OH})_{2}\right]$, comunemente definito calcitriolo.

La fonte principale di vitamina $D$ risiede nella sintesi endo- 


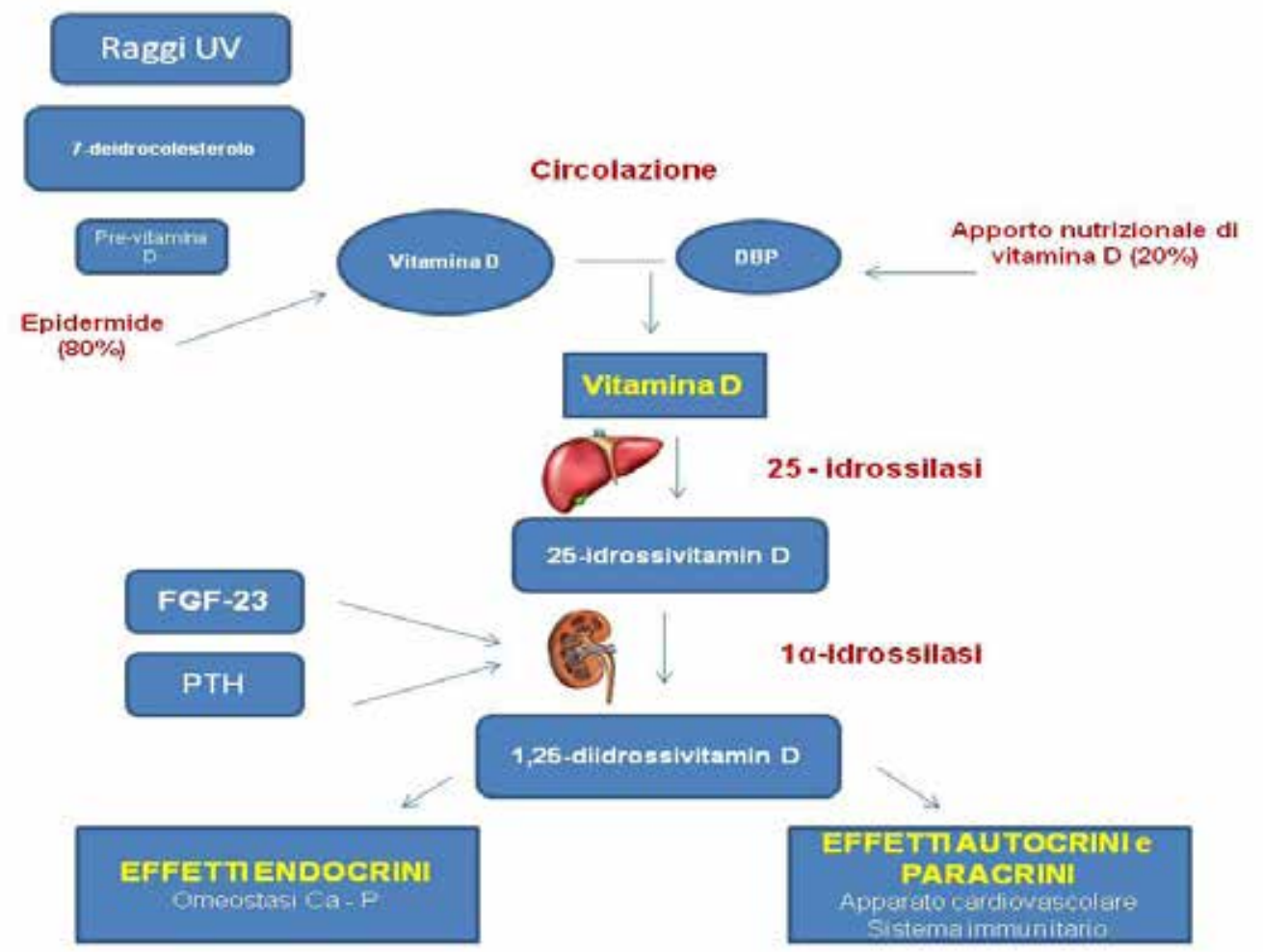

Fig. 1 - Metabolismo della vitamina $\mathrm{D}$.

gena a livello cutaneo (Fig. 1) grazie all'esposizione ai raggi ultravioletti (UVB) che permettono la conversione del precursore 7-deidrocolesterolo (prodotto a livello epatico) in pre-vitamina $D$ e, quindi, nell'isomero definitivo vitamina D; il cibo rappresenta una fonte minore di vitamina $D(6)$.

La vitamina $D$ esiste in due isoforme, colecalciferolo (vitamina $D_{3}$ ), sintetizzato a livello cutaneo e contenuto prevalentemente in cibi animali come il pesce e i suoi derivati, ed ergocaliferolo (vitamina $D_{2}$ ), che non può essere sintetizzato dall'uomo ma che è contenuto in diverse specie di funghi commestibili, anche se presenta una minore biodisponibilità rispetto alla vitamina $D_{3}$.

La vitamina $D$ viene trasportata nel torrente circolatorio grazie alla glicoproteina "vitamin D-binding protein" (DBP) secreta a livello epatico (7), dove avviene anche la prima idrossilazione della vitamina stessa a 25 -idrossivitamina D [25(OH)D] (Fig. 1), il cui dosaggio ematico consente di stabilire l'assetto della stessa, in quanto rappresenta la principale isoforma plasmatica, e la quantità introdotta con l'alimentazione (6).

La successiva modificazione enzimatica avviene a livello renale con un secondo processo di idrossilazione che porta alla formazione di 1.25 diidrossi vitamina $D\left[1.25(\mathrm{OH})_{2} \mathrm{D}\right]$, la forma attiva della vitamina. La produzione della forma attiva avviene sotto lo stretto controllo del metabolismo osseo ed è, per esempio, stimolata dall'ormone paratiroideo (PTH) e inibita dal fibroblast growth factor-23 (FGF-23) allo scopo di mantenere livelli adeguati di calcemia.
Gli effetti biologici dell' $\left[1.25(\mathrm{OH})_{2} \mathrm{D}\right]$ sono di tipo sia autocrino che paracrino, in quanto l'espressione dell'enzima $1 \alpha$-idrossilasi (che catalizza la seconda reazione di idrossilazione a livello renale producendo la forma attiva della vitamina $D$ ) è stata documentata anche a livello di tessuti extrarenali come, per esempio, l'apparato cardiovascolare (10). Inoltre, I'[1.25(OH) 2 D] esercita i suoi effetti biologici dopo essersi legato al proprio recettore (VDR) ubiquitariamente distribuito $(3,8,9)$.

II catabolismo dell'[1.25(OH) 2 D] è affidato, in prima battuta, a un processo di 24-idrossilazione indotto dalla stessa attivazione del VDR, allo scopo di scongiurare un'ipotetica intossicazione da vitamina D (Fig. 1).

\section{I livelli ideali di vitamina D}

Per quanto concerne i livelli di riferimento di vitamina D ottenibili grazie all'intake dietetico, questi sono basati soprattutto sugli effetti benefici esercitati dalla vitamina stessa in merito alla prevenzione di rachitismo, osteomalacia e fratture patologiche (10-13). Un evidente deficit di vitamina $D$ determina una riduzione dell'assorbimento a livello intestinale con conseguente ipocalcemia. La riduzione dei livelli di calcemia stimola, grazie alla sensibilizzazione dei cosiddetti CaSR (calcium sensing receptors) la produzione di PTH da parte delle ghiandole paratiroidee, con lo scopo di mantenere adeguati livelli di calcio plasmatico mediante l'aumentato rilascio a livello osseo e il maggior riassorbimento a livello 


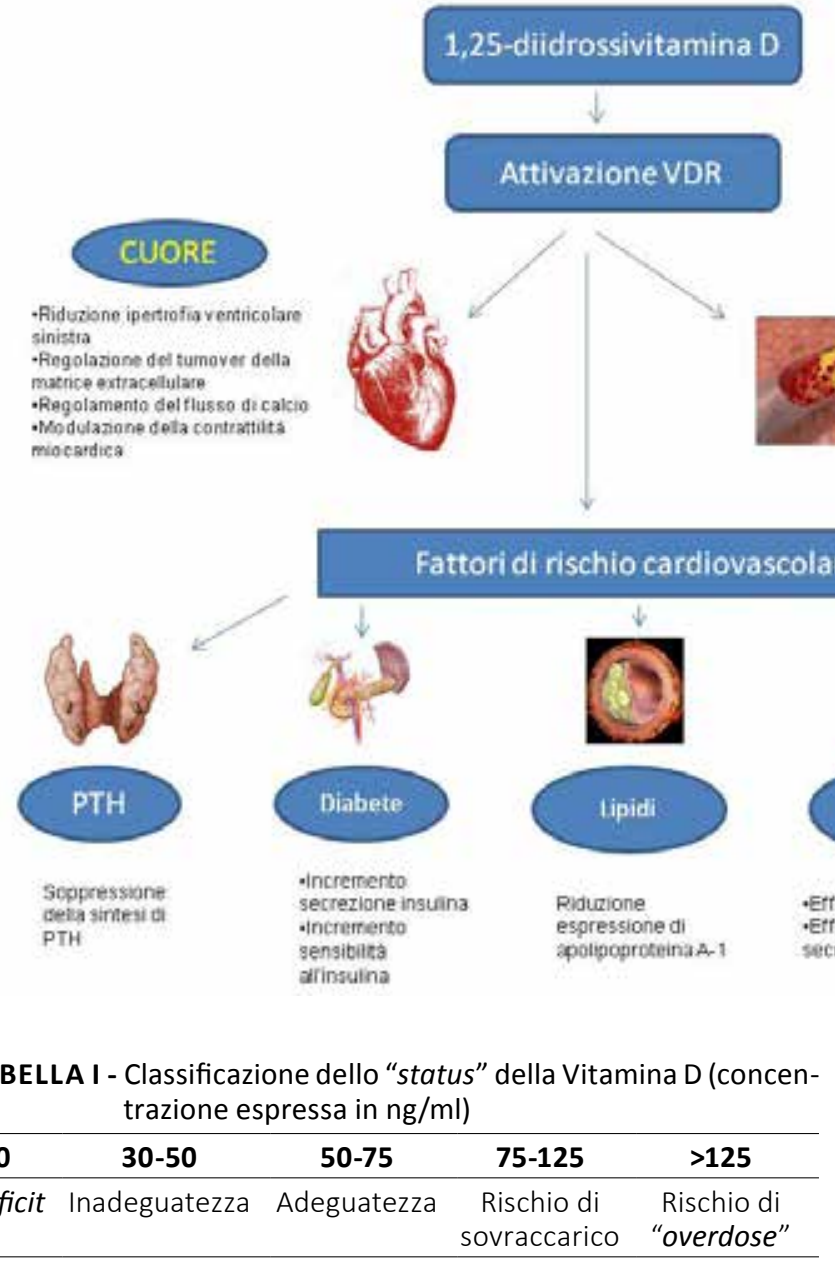

renale (elevati livelli di PTH si associano, generalmente, a basse concentrazioni di vitamina $D$ ).

In letteratura, sono stati proposti diversi valori di cut-off per quanto concerne i livelli di vitamina D; un report del 2011 evidenziava come una concentrazione sierica di $20 \mathrm{ng} / \mathrm{mL}$ di 25(OH)D fosse appropriata per l'ottenimento dei livelli richiesti nel $97.5 \%$ della popolazione generale (12) (Tab. I).

Allo stato attuale, diversi studi epidemiologici hanno evidenziato come, nella popolazione generale, vi sia un "inta$k e^{\prime \prime}$ generale (dieta più supplementi) di vitamina $\mathrm{D}$ inferiore a $200 \mathrm{UI} / d i e$ con il risultato di ottenere un sostanziale deficit della stessa $(14,15)$.

Per ottenere le concentrazioni desiderate di vitamina $D$ di $20 \mathrm{ng} / \mathrm{mL}$ sarebbe necessaria un'introduzione di almeno $800 \mathrm{UI} /$ die nel periodo invernale in Europa e di 600-800 UI/ die in presenza di scarsa (o assente) esposizione ai raggi solari (12).

Trial randomizzati e metanalisi hanno evidenziato come la supplementazione di vitamina D (nella misura di 800$2000 \mathrm{UI} /$ die) possa ridurre l'incidenza di fratture (16).

La terapia con vitamina $D$ è, inoltre, standardizzata nei
Fig. 2 - Effetti cardiovascolari secondari all'attivazione del recettore della vitamina $D(V D R)$.

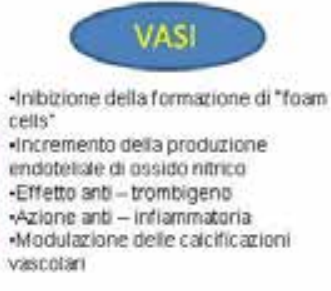

\section{VASI}

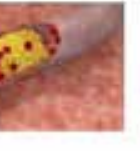

- Inbitione della formatione di "foam

- incremento dela produzion

- Ertetro snb - trombigeno

Azione ant-irdiammatori viescotian

TABELLA I - Classificazione dello “status" della Vitamina D (concen-
trazione espressa in ng/ml)
\begin{tabular}{lcccc}
\hline $\mathbf{3 0}$ & $\mathbf{3 0 - 5 0}$ & $\mathbf{5 0 - 7 5}$ & $\mathbf{7 5 - 1 2 5}$ & $>\mathbf{1 2 5}$ \\
\hline Deficit & Inadeguatezza & Adeguatezza & $\begin{array}{c}\text { Rischio di } \\
\text { sovraccarico }\end{array}$ & $\begin{array}{c}\text { Rischio di } \\
\text { "overdose" }\end{array}$ \\
\hline
\end{tabular}

pazienti affetti da osteoporosi (17) ma i meccanismi fisiopatologici che stanno alla base della riduzione del rischio fratturativo non sono stati del tutto chiariti, in quanto la terapia supplementare con vitamina $D$ non sembra eserciti effetti significativi a livello di densità ossea (18).

Allo stato attuale, non vi è un consenso univoco sulla dose di vitamina $D$ che andrebbe impiegata nei pazienti affetti da osteoporosi e in merito a quali livelli di $25(\mathrm{OH}) \mathrm{D}$ dovrebbero essere raggiunti (19-23).

I supplementi di vitamina $D$ (colecalciferolo o ergocalciferolo) determinano un incremento dei livelli di $25(\mathrm{OH}) \mathrm{D}$ in maniera dose-dipendente: $1000 \mathrm{UI} /$ die di vitamina D determinano un incremento di circa $10 \mathrm{ng} / \mathrm{mL}$ dei livelli di $25(\mathrm{OH})$ D (19-23).

\section{Vitamina D e malattia cardiovascolare}

Partendo dagli anni '30 e '40, anni in cui si diffuse a macchia d'olio l'impiego di prodotti contenenti vitamina D (latte e derivati, per esempio), fu subito evidente una riduzione dell'incidenza dei casi di rachitismo e l'eradicazione quasi totale, almeno in Europa e negli Stati Uniti, dei deficit di vitamina D nei bambini e negli adolescenti (24). A fare da contraltare, già dai primi anni ' 50 , un aumento dell'incidenza dei casi di ipercalcemia e di valvulopatie cardiache (in prevalenza stenosi aortiche sopravalvolari) in giovani adolescenti (24).

In realtà molti dei casi succitati furono poi ricondotti alle manifestazioni cliniche della sindrome di Williams, una pato- 
logia ereditaria legata al cromosoma X. Allo stesso tempo, però, diversi studi sperimentali negli animali hanno evidenziato come dosaggi standard di vitamina $\mathrm{D}$ possano indurre calcificazioni vascolari, soprattutto in caso di deficit della funzione renale $(25,26)$.

Nei primi anni ' 80 fu notato come l'aumento dell'incidenza di eventi cardiovascolari fosse particolarmente evidente nei mesi invernali (27); questa osservazione diede l'inizio dapprima a una serie di speculazioni e, poi, all'avvio di una serie di lavori sperimentali di ricerca che hanno raggiunto il culmine negli ultimi 10 anni (28-30).

Allo scopo di analizzare gli effetti molecolari della vitamina $D$ a livello dell'apparato cardiovascolare, risulta didatticamente utile analizzare cosa avviene, singolarmente, a livello di fattori di rischio cardiovascolare, nei vasi arteriosi e nel tessuto miocardico (Fig. 2).

\section{Vitamina D e fattori di rischio cardiovascolare}

Come descritto in precedenza, la vitamina $D$ esercita $i$ suoi effetti grazie all'interazione con recettori specifici, i VDR, la cui attivazione esercita un ruolo inibitorio sulla sintesi di PTH (8). Elevate concentrazioni di ormone paratiroideo sono, a loro volta, associate a evidenti fattori di rischio cardiovascolare (malattia renale cronica, obesità) e a un rischio maggiore di eventi cardiovascolari (31).

Studi sperimentali hanno evidenziato come il PTH favorisca la deposizione di calcio all'interno dei cardiomiociti, generi stress ossidativo, possieda effetti pro-aritmici, induca ipertrofia miocardica e disfunzione endoteliale e, infine, favorisca la secrezione di aldosterone (32-34).

Riguardo all'azione esercitata dalla vitamina D sul sistema renina-angiotensina-aldosterone (RAAS), la soppressione di quest'ultimo potrebbe essere alla base delle proprietà antiipertensive della vitamina stessa (35). La spiegazione potrebbe risiedere nell'attivazione del VDR, in grado, a sua volta, di sopprimere la trascrizione dell'mRNA per la renina (35). Studi sperimentali hanno evidenziato come il deficit di vitamina D (al netto di ipocalcemia e iperPTH) possa contribuire alla genesi di uno stato ipertensivo dovuto all'incremento delle resistenze vascolari e alla conseguente vasocostrizione (36).

L'attivazione del VDR sembra, inoltre, fare da apripista a un effetto anti-diabetico della vitamina $D$, ivi compresa un'azione favorente la secrezione e la sensibilità all'insulina; allo stesso tempo, l'attivazione del VDR comporta l'inattivazione di diverse citochine in grado di determinare l'apoptosi delle cellule $\beta$ del pancreas $(37,38)$.

Meno chiari sono i potenziali effetti esercitati dai livelli di vitamina $D$ sul metabolismo lipidico (39-41). Sembra, comunque, ben documentato come l'attivazione del VDR sia in grado di sopprimere l'espressione dell'apolipoproteina A1, la principale componente del colesterolo HDL e come, allo stesso tempo, potrebbe indirettamente incrementare l'assorbimento di calcio a livello intestinale con riduzione della formazione dei complessi calcio-lipidi successivamente eliminati con le feci (39-41). A far da contraltare a quanto appena esposto, un'ipotesi alternativa prevede che l'aumentato assorbimento di calcio a livello intestinale indotto dalla vitamina $D$ potrebbe ridurre i livelli di trigliceridi riducendone la formazione a livello epatico $(39,40)$. Ulteriori effetti benefici della vitamina $D$ nei confronti del metabolismo lipidico potrebbero essere mediati dalla riduzione dei livelli di PTH (in grado di favorire la lipolisi) e dall'aumento della secrezione di insulina $(39,40)$.

\section{Vitamina D e vasi arteriosi}

Gli effetti molecolari esercitati dalla vitamina $D$ a carico dei vasi arteriosi sono ben noti e diffusamente studiati (42).

Studi sperimentali suggeriscono come la vitamina $D$ abbia caratteristiche tali da contrastare la progressione del danno aterosclerotico inibendo, in primis, la trasformazione dei macrofagi in cellule schiumose (meglio conosciute con il nome di "foam cells") (43). La forma attiva della vitamina D è in grado di favorire la riparazione del danno endoteliale inducendo la secrezione del VEGF (vascular endothelial growth factor) a livello delle cellule muscolari lisce della parete vasale.

Un ulteriore effetto miorilassante, esercitato dal VDR, è collegato all'aumentata espressione del recettore di tipo B dell'endotelina (EDNRB) e alla ridotta espressione della Oxytocine receptor (OXTR).

Ulteriori proprietà anti-aterogene collegate all'attivazione del VDR sono in relazione con l'attività anti-infiammatoria come evidenziato dalla riduzione della secrezione di interleuchina 6 (IL-6) da parte delle cellule endoteliali e dei macrofagi e dall'aumentata produzione di ossido nitrico (42).

L'attivazione del VDR è, inoltre, in grado, di promuovere la riparazione del danno endoteliale mediata dalle cellule della linea mieloide, stimolando la produzione di SDF-1 (stromal cell-derived factor) (44).

A livello dei vasi arteriosi, un aspetto di particolare importanza clinica è rappresentato dall'effetto giocato dalla vitamina $D$ sulla formazione e sulla relativa progressione delle calcificazioni vascolari. Sia la carenza che l'eccesso di vitamina D possono favorire la trasformazione in senso osteoblastico delle cellule della muscolatura liscia delle pareti vasali (42). II sovraccarico di fosforo, in grado di stimolare il catabolismo della vitamina $D$ grazie allo stimolo dell'attività della 24idrossilasi, è un punto cardine nella genesi delle calcificazioni vascolari (24). L'attivazione dei VDR favorisce l'assorbimento di fosforo (e il conseguente aumento della fosforemia), stimolando la secrezione di FGF-23 a livello osteocitario con il successivo aumento dell'escrezione urinaria di fosfato. Allo stesso tempo, l'attivazione dei VDR determina un aumento dell'espressione di klotho, un recettore in grado di lavorare in simbiosi con FGF-23 per prevenire la formazione delle calcificazioni vascolari (42). In 
ultima analisi, la formazione delle calcificazioni vascolari sembra essere strettamente dipendente dalle interazioni tra vitamina D, klotho e FGF-23 (42).

\section{Vitamina D e cuore}

Recettori per la vitamina D sono presenti a livello di cardiomiociti e cellule fibroblastiche e la loro presenza è stata associata a ipertrofia miocardica in topi knockout per il gene VDR (44).

Studi effettuati su cavie hanno evidenziato come il deficit di vitamina D si associ a ipertrofia ventricolare e fibrosi interstiziale a livello dei cardiomiociti e del tessuto di conduzione miocardico (44). Ciò che risulta interessante è che il quadro di ipertrofia miocardica è accompagnato da un'aumentata espressione miocardica del gene e della proteina finale del VDR $(44,45)$

In studi sperimentali, l'attivazione del VDR a livello miocardico sembra essere in grado di modulare il turnover dei componenti della matrice extracellulare regolando l'espressione delle metalloproteinasi $(44,45)$.

In aggiunta, l'attivazione del VDR influenza la contrattilità del tessuto miocardico, agendo, probabilmente, sul flusso di calcio transmembrana e, quindi, sul potenziale d'azione delle cellule miocardiche $(44,45)$.

Gli effetti della vitamina D sulle capacità contrattili del tessuto miocardico sono ancora poco noti e ancora oggetto di studio; tuttavia, I'1-25(OH) 2 è in grado di agire sulla fase di ripolarizzazione delle cellule del tessuto di conduzione miocardico, a indicare un ruolo del VDR sulla funzione diastolica $(44,45)$.

\section{Vitamina D, apparato cardiovascolare e studi clinici: cosa imparare dall'analisi della letteratura scientifica}

\section{Gli studi osservazionali}

Sono molteplici gli studi osservazionali che hanno documentato un'associazione tra livelli di vitamina D (in termini di concentrazioni di 25(OH)D) e rischio cardiovascolare, ma risentono tutti di alcuni bias di fondo. In primo luogo, i livelli di $25(\mathrm{OH}) \mathrm{D}$ possono essere sottostimati in soggetti con attività fisica limitata (con conseguente riduzione dell'esposizione ai raggi solari), in soggetti obesi (per la deposizione di vitamina $\mathrm{D}$ a livello del tessuto adiposo) e in coloro i quali presentano un quadro di infiammazione cronica di grado severo (per aumentata espressione dell'enzima 24-idrossilasi) $(46,47)$. Inoltre, alcuni Autori hanno postulato come il deficit di vitamina $D$ possa essere considerato un marker di ridotto benessere fisico, ipotesi supportata dal fatto che il deficit di vitamina $D$ è associato a diverse patologie ad andamento cronico (46).

Sono stati segnalati diversi casi di rachitismo associati a cardiomiopatie di diversa natura in bambini con deficit di vitamina $D$ e ipocalcemia; in questi pazienti, il trattamento con vitamina $D$ e la supplementazione di calcio determinavano sia un miglioramento dal punto di vista clinico generale che un effetto benefico sul ventricolo sinistro, sia dal punto di vista anatomico che da quello funzionale (48-50).

In una metanalisi di studi prospettici di coorte, per un totale di 283.537 pazienti di cui 55.816 incidenti per ipertensione arteriosa, si è evidenziato un hazard ratio di 0.88 per un incremento, rispetto al livello basale, di $10 \mathrm{ng} / \mathrm{mL}$ di 25(OH)D (51).

Altre metanalisi hanno, d'altro canto, evidenziato come bassi livelli sierici di $25(\mathrm{OH}) \mathrm{D}$ siano associati a un rischio maggiore di nuovi casi di diabete mellito di tipo 2 e di diabete gestazionale (52-56).

È stata, inoltre, osservata un'associazione significativa tra deficit di vitamina $\mathrm{D}$ e obesità, anche se i dati descritti in letteratura sono ancora troppo scarsi per poter avere un impatto degno di nota (57).

Una maggior mole di dati di letteratura evidenzia un forte legame tra deficit di vitamina $D$ e colesterolo HDL, a testimonianza di un aumentato rischio aterogeno in questa popolazione di pazienti (58).

Sempre da studi di coorte è emerso come il deficit di vitamina D sia significativamente associato ad altri fattori di rischio cardiovascolare, come infiammazione, malattia renale cronica e anemia $(59,60)$.

Entrando nel campo della patologia cardiovascolare (e non solo dei fattori di rischio) associata al deficit di vitamina $D$, sono diverse le metanalisi di studi prospettici che hanno documentato un aumento dell'incidenza di eventi cardiovascolari maggiori e di mortalità per cause cardiovascolari associate alla diminuzione dei livelli sierici di 25(OH)D (61-64).

Sebbene le metanalisi e la maggior parte degli studi di popolazione abbiano evidenziato un'associazione tra eventi cardiovascolari maggiori e deficit di vitamina $\mathrm{D}$, una quota parte di studi clinici ha posto l'attenzione anche sulla correlazione tra patologia cardiovascolare e livelli elevati di vitamina $D$ (65-68); in quest'ultimo caso vanno esaminati in maniera distinta i soggetti arruolati in quanto i livelli aumentati di vitamina $D$ potrebbero derivare sia da una modificazione dello stile di vita (maggiore esposizione ai raggi solari) che da un carico supplementare di vitamina D.

Sostanzialmente si può affermare che, nel caso di livelli di vitamina $D$ superiori a quelli normali, si rendono necessari ulteriori valutazioni di tipo prospettico e randomizzato; allo stesso tempo, si può, comunque, affermare come condizioni di intossicazioni da vitamina $D$ con ipercalcemia si verifichino solo con valori di $25(\mathrm{OH}) \mathrm{D}$ superiori a $150 \mathrm{ng} /$ $\mathrm{mL}$, condizione che può essere raggiunta solo nel caso di "overdose" da vitamina D.

Nell'ambito delle patologie cardiovascolari associate a bassi livelli di $25(\mathrm{OH}) \mathrm{D}$, un legame è stato riscontrato con la cardiopatia ischemica e l'infarto del miocardio (66-68) pur in presenza di reperti quasi normali alla coronarografia ov- 
vero al Doppler dei vasi epiaortici $(69,70)$.

Bassi livelli di 25(OH)D sono stati, inoltre, associati alla presenza di disfunzione endoteliale e di malattia vascolare periferica (71).

Ulteriori metanalisi di studi prospettici hanno evidenziato un rischio decisamente aumentato di stroke e altri eventi cerebrovascolari in presenza di livelli deficitari di vitamina D $(72,73)$.

Tirando le somme dei dati provenienti da studi di tipo osservazionale, si può concludere asserendo che basse concentrazioni di vitamina $D$ (al di sotto di $20 \mathrm{ng} / \mathrm{mL}$ ) rappresentano un marker di aumentato rischio cardiovascolare, in particolare nei pazienti affetti da malattia renale cronica nei quali livelli particolarmente bassi di vitamina $D$ attiva si associano anche a un aumento del tasso di mortalità. Nei pazienti affetti da malattia renale cronica, inoltre, la supplementazione con vitamina $D$ attiva (calcitriolo o analoghi) è associata a un miglioramento dei tassi di sopravvivenza (74).

\section{I trial randomizzati controllati (RCT)}

Per quanto concerne l'associazione tra livelli di vitamina $\mathrm{D}$ e fattori di rischio cardiovascolare, alcuni RCT e diverse metanalisi di RCT hanno suggerito la possibilità di un ruolo anti-ipertensivo della vitamina $D(75,76)$. D'altro canto, una metanalisi del 2015 (4541 pazienti) ha concluso per la mancanza di effetti significativi, da parte della vitamina $\mathrm{D}$, sulla pressione arteriosa (77). Tra l'altro, questi ultimi dati sono stati confermati da un RCT condotto su circa 200 pazienti ipertesi, nei quali la supplementazione con vitamina $D$ non ha sortito alcun effetto sui livelli di pressione arteriosa (78).

Rimanendo nell'ambito dei fattori di rischio cardiovascolare, una metanalisi di RCT, condotta su circa 43000 pazienti, ha analizzato l'effetto della terapia con vitamina $D$ sui parametri di omeostasi glucidica (secrezione e resistenza insulinica, livelli di $\mathrm{HbA1c}$ ), evidenziando come la supplementazione di 25(OH)D non influisca sui parametri succitati (79).

Dall'analisi di ulteriori RCT è stato anche possibile evidenziare l'assoluta mancanza di effetti evidenti della terapia con vitamina $D$ sui parametri del metabolismo lipidico $(80,81)$ e su quelli di infiammazione cronica, come sottolineato dagli scarsi effetti sui livelli di proteina C-reattiva (PCR) (82-84).

Alcuni RCT sembrano assegnare un ruolo di immunomodulazione alla vitamina $D$, come evidenziato dall'incremento del numero di cellule $\mathrm{T}$ in risposta alla terapia con 25(OH)D (85).

Passando ai rapporti tra vitamina $\mathrm{D}$ e patologia cardiovascolare, in primo luogo va detto che alcune metanalisi di RCT hanno evidenziato uno scarso effetto della supplementazione con vitamina D sulla disfunzione endoteliale (86-88), mentre altre hanno dimostrato gli scarsi effetti sui parametri di "stiffness" (pulse wave velocity e augmentation index).

Per quanto concerne gli effetti della vitamina $D$ nei pazienti affetti da scompenso cardiaco, una metanalisi di 7 RCT (573 pazienti) ha escluso effetti significativi della supplemen- tazione con vitamina $\mathrm{D}$ sul valore della frazione d'eiezione (FE) del ventricolo sinistro e sui livelli dei peptidi natriuretici (83). Al contrario, lo studio VINDICATE del 2016 ha evidenziato un effetto positivo della terapia con vitamina $D$ in pazienti scompensati con un aumento di circa il $6 \%$ del valore di FE rispetto alla popolazione trattata con placebo e una riduzione dei volumi telesistolico e telediastolico del ventricolo sinistro (89).

Andando ad analizzare i RCT disegnati in primo luogo per evidenziare gli effetti della supplementazione con vitamina $D$ sui parametri del metabolismo minerale, si è documentata l'assenza di effetti significativi in termini di eventi e di mortalità cardiovascolare $(90,91)$, soprattutto in quei trial nei quali non era prevista la terapia con calcio, di per sé associata a un aumentato rischio di infarto del miocardio (92).

Anche dall'analisi di diversi RCT, risulterebbe evidente come la supplementazione con vitamina $D$ non eserciti effetti sull'incidenza di infarto miocardico e/o cardiopatia ischemica cronica, così come su quella di eventi cerebrovascolari (93).

In definitiva, dall'analisi dei diversi RCT emerge un ruolo in chiaroscuro per quanto riguarda gli effetti della terapia con vitamina $D$ sulla malattia cardiovascolare; ad ogni modo $i$ pur evidenziati effetti sull'apparato cardiovascolare appaiono comunque di impatto limitato rispetto a quanto prodotto da molecole come ACE-inibitori o statine.

\section{Conclusioni}

Le attuali evidenze prodotte dalla letteratura scientifica, in buona sostanza, testimoniano gli scarsi effetti prodotti dalla terapia con vitamina $\mathrm{D}$ sul rischio cardiovascolare.

Ciò che andrà studiato nei futuri RCT sarà l'impatto della supplementazione con vitamina $D$ sui fattori di rischio cardiovascolare in quei pazienti con grave deficit di $25(\mathrm{OH}) \mathrm{D}$ e, soprattutto, andranno studiati quali sono i livelli potenzialmente tossici della vitamina stessa (94).

A questo proposito, gli effetti protettivi, in termini di patologia fratturativa, della vitamina $D$ sono stati documentati per dosi giornaliere di vitamina D di 800-2000 UI, con un limite superiore di $4000 \mathrm{UI}$, prima che possano comparire effetti collaterali e/o tossici legati a ipercalcemia (95).

Per quanto riguarda la periodicità della misurazione dei livelli di $25(\mathrm{OH}) \mathrm{D}$, il dibattito è aperto tra coloro i quali sono fautori di un dosaggio periodico a tappeto e coloro i quali, al contrario, pongono l'indicazione a un controllo seriato solo nei pazienti affetti da patologie in grado di indurre deficit di vitamina $\mathrm{D}$ (vedi la popolazione dei pazienti affetti da malattia renale cronica) (96).

Un consenso piuttosto unanime esiste sul fatto che vadano evitati livelli di $25(\mathrm{OH}) \mathrm{D}$ inferiori a $20 \mathrm{ng} / \mathrm{mL}$, livelli per i quali è necessario iniziare la terapia supplementare con vitamina $D(6,11-15,19)$. 


\section{Disclosures}

Financial support: No financial support was received for this submission.

Conflict of interest: The authors have no conflict of interest.

\section{Bibliografia}

1. Holick MF. McCollum Award Lecture, 1994: vitamin D -- new horizons for the $21^{\text {st }}$ century. Am J Clin Nutr. 1994;60:619-30.

2. Bouillon R, Suda T. Vitamin D: calcium and bone homeostasis during evolution. Bonekey Rep. 2014;3:480.

3. Bouillon R. Vitamin D and human health: lessons from vitamin D receptor null mice. Endocr Rev. 2008;29:726-76.

4. Pludowski P. Vitamin D effects on musculoskeletal health, immunity, autoimmunity, cardiovascular disease, cancer, fertility, pregnancy, dementia and mortality -- a review of recent evidence. Autoimmun Rev. 2013;12:976-89.

5. Rosen CJ. The nonskeletal effects of vitamin D: an Endocrine Society scientific statement. Endocr Rev. 2012;33:456-92.

6. Holick MF. Vitamin D deficiency. N Engl J Med. 2007;357:266-81.

7. Delanghe JR, Speeckaert R, Speeckaert MM. Behind the scenes of vitamin $D$ binding protein: more than vitamin $D$ binding. Best Pract Res Clin Endocrinol Metab. 2015;29:773-86.

8. Christakos S, Dhawan P, Verstuyf A, Verlinden L, Carmeliet G. Vitamin D: metabolism, molecular mechanism of action, and pleiotropic effects. Physiol Rev. 2016;96:365-408.

9. Wang $Y$, DeLuca HF. Is the vitamin D receptor found in muscle? Endocrinology. 2011;152:354-63.

10. Ross AC. The 2011 report on dietary reference intakes for calcium and vitamin D from the Institute of Medicine: what clinicians need to know. J Clin Endocrinol Metab. 2011;96:53-8.

11. Cashman KD, Kiely M. Recommended dietary intakes for vitamin D: where do they come from, what do they achieve and how can we meet them? J Hum Nutr Diet. 2014;27:434-42.

12. German Nutrition Society. New reference values for vitamin D. Ann Nutr Metab. 2012;60:241-6.

13. Cashman KD. Vitamin D: dietary requirements and food fortification as a means of helping achieve adequate vitamin $D$ status. J Steroid Biochem Mol Biol. 2015;148:19-26.

14. Calvo MS, Whiting SJ, Barton CN. Vitamin D intake: a global perspective of current status. J. Nutr. 2005;135:310-6.

15. Wahl DA. A global representation of vitamin $D$ status in healthy populations. Arch Osteoporos. 2012;7:155-72.

16. Bischoff-Ferrari HA. A pooled analysis of vitamin D dose requirements for fracture prevention. N Engl J Med. 2012;367: 40-9.

17. Kanis JA. European guidance for the diagnosis and management of osteoporosis in postmenopausal women. Osteoporos Int. 2013;24:23-57.

18. Reid IR, Bolland MJ, Grey A. Effects of vitamin D supplements on bone mineral density: a systematic review and metaanalysis. Lancet. 2014;383:146-55.

19. Holick MF. Evaluation, treatment, and prevention of vitamin D deficiency: an Endocrine Society clinical practice guideline. J Clin Endocrinol Metab. 2011;96:1911-30.

20. Rosen CJ. IOM committee members respond to Endocrine Society vitamin D guideline. J Clin Endocrinol Metab. 2012;97: 1146-52.

21. Holick MF. Guidelines for preventing and treating vitamin D deficiency and insufficiency revisited. J Clin Endocrinol Metab. 2012;97:1153-8.

22. Brouwer-Brolsma EM. Vitamin D: do we get enough? A discussion between vitamin $D$ experts in order to make a step towards the harmonisation of dietary reference intakes for vitamin D across Europe. Osteoporos Int. 2013;24:1567-77.

23. Reid IR, Bolland MJ. Skeletal and nonskeletal effects of vitamin $\mathrm{D}$ : is vitamin $\mathrm{D}$ a tonic for bone and other tissues? Osteoporos Int. 2014;25:2347-57.

24. Zittermann A. Vitamin D and cardiovascular disease. Anticancer Res. 2014;34:4641-8.

25. Kummerow FA. Nutrition imbalance and angiotoxins as dietary risk factors in coronary heart disease. Am J Clin Nutr. 1979;32:58-83.

26. Drüeke TB, Massy ZA. Role of vitamin D in vascular calcification: bad guy or good guy? Nephrol Dial Transplant. 2012;27:1704-7.

27. Scragg R. Seasonality of cardiovascular disease mortality and the possible protective effect of ultra-violet radiation. Int J Epidemiol. 2012;10:337-41.

28. Beveridge LA, Witham MD. Vitamin $D$ and the cardiovascular system. Osteoporos Int. 2013;24:2167-80.

29. Pilz S, Gaksch M, O'Hartaigh B, Tomaschitz A, März W. The role of vitamin $D$ deficiency in cardiovascular disease: where do we stand in 2013? Arch Toxicol. 2013;87:2083-103.

30. Carvalho LS, Sposito AC. Vitamin D for the prevention of cardiovascular disease: are we ready for that? Atherosclerosis. 2015;241:729.40.

31. Van Ballegooijen AJ, Reinders I, Visser $M$, Brouwer IA Parathyroid hormone and cardiovascular disease events: a systematic review and meta-analysis of prospective studies. Am Heart J. 2013;165:655-64.

32. Fitzpatrick LA, Bilezikian JP, Silverberg SJ. Parathyroid hormone and the cardiovascular system. Curr Osteoporos Rep. 2008;6: 77-83.

33. Rutledge MR. Parathyroid hormone, a crucial mediator of pathologic cardiac remodeling in aldosteronism. Cardiovasc Drugs Ther. 2013;27:161-70.

34. Tomaschitz A Aldosterone and parathyroid hormone interactions as mediators of metabolic and cardiovascular disease. Metabolism. 2014;63:20-31.

35. Yuan W. 1,25-dihydroxyvitamin D3 suppresses renin gene transcription by blocking the activity of the cyclic AMP response element in the renin gene promoter. J Biol Chem. 2007;282:29821-30.

36. Chen S, Sun Y, Agrawal DK. Vitamin D deficiency and essential hypertension. J Am Soc Hypertens. 2015;9:885-901.

37. Mathieu C. Vitamin D and diabetes: where do we stand? Diabetes Res Clin Pract. 2015;108:201-9.

38. Pilz S. Role of vitamin $D$ in the development of insulin resistance and type 2 diabetes. Curr Diab Rep. 2013;13:261-70.

39. Jorde R, Grimnes G. Vitamin D and metabolic health with special reference to the effect of vitamin $D$ on serum lipids. Prog Lipid Res. 2011;50:303-12.

40. Jaimungal $S$, Wehmeier $K$, Mooradian AD, Haas MJ. The emerging evidence for vitamin $D$-mediated regulation of apolipoprotein A-I synthesis. Nutr Res. 2011;31:805-12.

41. Wehmeier K. Inhibition of apolipoprotein Al gene expression by 1, 25-dihydroxyvitamin D3. Biochim Biophys Acta. 2005; 1737:16-26.

42. Norman PE, Powell JT. Vitamin D and cardiovascular disease. Circ Res. 2014;114:379-93.

43. Oh J. $1,25(\mathrm{OH}) 2$ vitamin $\mathrm{D}$ inhibits foam cell formation and suppresses macrophage cholesterol uptake in patients with type 2 diabetes mellitus. Circulation. 2009;120:687-98.

44. Gardner DG, Chen S, Glenn DJ. Vitamin D and the heart. Am J Physiol Regul Integr Comp Physiol. 2013;305:R969-77. 
45. Pilz S, Tomaschitz A, Drechsler C, Dekker JM, März W. Vitamin $D$ deficiency and myocardial diseases. Mol Nutr Food Res. 2010;54:1103-13.

46. Autier P, Boniol M, Pizot C, Mullie P. Vitamin D status and ill health: a systematic review. Lancet Diabetes Endocrinol. 2014;2:76-89.

47. Reid $D$. The relation between acute changes in the systemic inflammatory response and plasma 25-hydroxyvitamin D concentrations after elective knee arthroplasty. Am J Clin Nutr. 2011;93:1006-11.

48. Maiya S. Hypocalcaemia and vitamin D deficiency: an important, but preventable, cause of life-threatening infant heart failure. Heart. 2008;94:581-4.

49. Pedrosa C, Ferraria N, Limbert C, Lope L. Hypovitaminosis D and severe hypocalcaemia: the rebirth of an old disease. BMJ Case Rep. http://dx.doi.org/10.1136/bcr2012007406 (2013).

50. Yilmaz O. Dilated cardiomyopathy secondary to ricketsrelated hypocalcaemia: eight case reports and a review of the literature. Cardiol Young. 2015;25:261-6.

51. Kunutsor SK, Apekey TA, Steur M. Vitamin D and risk of future hypertension: meta-analysis of 283,537 participants. Eur J Epidemiol. 2013;28:205-21.

52. Song Y. Blood 25-hydroxy vitamin D levels and incident type 2 diabetes: a meta-analysis of prospective studies. Diabetes Care. 2013;36:1422-8.

53. Zhang MX. Vitamin D deficiency increases the risk of gestational diabetes mellitus: a meta-analysis of observational studies. Nutrients. 2015;7:8366-75.

54. Khan $\mathrm{H}$, Kunutsor S, Franco $\mathrm{OH}$, Chowdhury R. Vitamin D, type 2 diabetes and other metabolic outcomes: a systematic review and meta-analysis of prospective studies. Proc Nutr Soc. 2013;72:89-97.

55. Ju SY, Jeong HS, Kim DH. Blood vitamin D status and metabolic syndrome in the general adult population: a dose-response meta-analysis. J Clin Endocrinol Metab. 2014;99:1053-63.

56. Dong JY. Vitamin D intake and risk of type 1 diabetes: a metaanalysis of observational studies. Nutrients. 2013;5:3551-62.

57. Pereira-Santos M, Costa PR, Assis AM, Santos CA, Santos DB. Obesity and vitamin D deficiency: a systematic review and meta-analysis. Obes Rev. 2015;16:341-9.

58. Kelishadi R, Farajzadegan Z, Bahreynian M. Association between vitamin $D$ status and lipid profile in children and adolescents: a systematic review and meta-analysis. Int J Food Sci Nutr. 2014;65:404-10.

59. Prietl B, Treiber G, Pieber TR, Amrein K. Vitamin D and immune function. Nutrients. 2013;5:2502-21.

60. Hewison M. An update on vitamin D and human immunity. Clin Endocrinol. 2012;76:315-25.

61. Fan H. Meta-analysis of circulating 25-hydroxyvitamin D levels and risk of cardiovascular and all-cause mortality in elderly population. Int J Cardiol. 2014;176:1025-9.

62. Chowdhury R. Vitamin D and risk of cause specific death: systematic review and meta-analysis of observational cohort and randomised intervention studies. BMJ. 2014;348:1903.

63. Theodoratou E, Tzoulaki I, Zgaga L, loannidis JP. Vitamin D and multiple health outcomes: review of systematic reviews and meta-analyses of observational studies and randomised trials. BMJ. 2014;348:2035.

64. Schöttker B. Vitamin D and mortality: meta-analysis of individual participant data from a large consortium of cohort studies from Europe and the United States. BMJ. 2014;348:3656.

65. Zittermann A. Vitamin D status and the risk of major adverse cardiac and cerebrovascular events in cardiac surgery. Eur
Heart J. 2013;34:1358-64.

66. Durup D. A reverse J-shaped association between serum 25-hydroxyvitamin $D$ and cardiovascular disease mortality: The CopD Study. J Clin Endocrinol Metab. 2015;100:2339-46.

67. Grant WB. Letter to the editor: the J-shaped 25-hydroxyvitamin $D$ concentration-cardiovascular disease mortality relation is very likely due to starting vitamin $D$ supplementation late in life. J Clin Endocrinol Metab. 2015;100:L49-50.

68. Schwarz P. Response to the letter by Grant. J Clin Endocrinol Metab. 2015;100:L51.

69. Degerud E. Vitamin D status was not associated with 'one-year' progression of coronary artery disease, assessed by coronary angiography in statin-treated patients. Eur J Prev Cardiol. 2015;22:594-602.

70. Alsancak Y. Relationship between serum vitamin D levels and angiographic severity and extent of coronary artery disease. Eur J Clin Invest. 2015;45:940-8.

71. Brøndum-Jacobsen P, Nordestgaard BG, Schnohr P, Benn M. 25-hydroxyvitamin $D$ and symptomatic ischemic stroke: an original study and meta-analysis. Ann Neurol. 2013;73:38-47.

72. Chowdhury R. Circulating vitamin D, calcium and risk of cerebrovascular disease: a systematic review and metaanalysis. Eur J Epidemiol. 2012;27:581-91.

73. Pilz S. Vitamin D supplementation: a promising approach for the prevention and treatment of strokes. Curr Drug Targets. 2011;12:88-96.

74. Zheng Z, Shi H, Jia J, Li D., Lin S. Vitamin D supplementation and mortality risk in chronic kidney disease: a meta-analysis of 20 observational studies. BMC Nephrol. 2013;14:199.

75. Moslehi N, Shab-Bidar S, Mirmiran P, Hosseinpanah F, Azizi F. Determinants of parathyroid hormone response to vitamin $D$ supplementation: a systematic review and meta-analysis of randomised controlled trials. Br J Nutr. 2015;114:1360-74.

76. Pilz S, Tomaschitz A, Ritz E, Pieber TR. Vitamin D status and arterial hypertension: a systematic review. Nat Rev Cardiol. 2009;6:621-30.

77. Beveridge LA. Effect of vitamin D supplementation on blood pressure: a systematic review and meta-analysis incorporating individual patient data. JAMA Intern Med. 2015;175:745-54.

78. Pilz S. Effects of vitamin D on blood pressure and cardiovascular risk factors: a randomized controlled trial. Hypertension. 2015;65:1195-201.

79. Seida JC. Clinical review: effect of vitamin D3 supplementation on improving glucose homeostasis and preventing diabetes: a systematic review and meta-analysis. J Clin Endocrinol Metab. 2014;99:3551-60.

80. Challoumas D. Vitamin D supplementation and lipid profile: what does the best available evidence show? Atherosclerosis. 2014;235:130-9.

81. Wang H, Xia N, Yang Y, Peng DQ. Influence of vitamin D supplementation on plasma lipid profiles: a meta-analysis of randomized controlled trials. Lipids Health Dis. 2012;11:42.

82. Chen N. Effect of vitamin D supplementation on the level of circulating high-sensitivity C-reactive protein: a meta-analysis of randomized controlled trials. Nutrients. 2014;6:2206-16.

83. Jiang WL. Vitamin D supplementation in the treatment of chronic heart failure: a meta-analysis of randomized controlled trials. Clin Cardiol. 2016;39:56-61.

84. Jamka M. The effect of vitamin D supplementation on selected inflammatory biomarkers in obese and overweight subjects: a systematic review with meta-analysis. Eur J Nutr. 2015. http:// dx.doi.org/ 10. 1007/ s00394-015-1089-5.

85. Bock $G$. The effect of vitamin $D$ supplementation on peripheral 
regulatory T cells and $\beta$ cell function in healthy humans: a randomized controlled trial. Diabetes Metab Res Rev. 2011; 27:942-5.

86. Stojanovic' $M$, Radenkovic' $M$. Vitamin $D$ versus placebo in improvement of endothelial dysfunction: a meta-analysis of randomized clinical trials. Cardiovasc Ther. 2015;33:145-54.

87. Joris PJ, Mensink RP. Effects of supplementation with the fatsoluble vitamins $E$ and $D$ on fasting flow-mediated vasodilation in adults: a meta-analysis of randomized controlled trials. Nutrients. 2015;7:1728-43.

88. Rodríguez AJ, Scott D, Srikanth V, Ebeling P. Effect of vitamin D supplementation on measures of arterial stiffness: a systematic review and meta-analysis of randomised controlled trials. Clin Endocrinol. 2016;84:645-57.

89. Witte KK. Effects of vitamin D on cardiac function in patients with chronic HF: The VINDICATE Study. J Am Coll Cardiol. 2016. http://dx.doi.org/ 10.1016/j.jacc.2016.03.508.

90. Bjelakovic G. Vitamin D supplementation for prevention of mortality in adults. Cochrane Database Syst Rev. 2014;1:CD007470.
91. Wang L, Manson JE, Song Y, Sesso HD. Systematic review: vitamin $D$ and calcium supplementation in prevention of cardiovascular events. Ann Intern Med. 2010;152:315-23.

92. Reid IR, Bristow SM, Bolland MJ. Calcium supplements: benefits and risks. J Intern Med. 2015;278:354-68.

93. Bolland MJ, Grey A, Gamble GD, Reid IR. The effect of vitamin D supplementation on skeletal, vascular, or cancer outcomes: a trial sequential meta-analysis. Lancet Diabetes Endocrinol. 2014;2:307-20.

94. Zittermann A, Prokop S, Gummert JF, Börgermann J. Safety issues of vitamin D supplementation. Anticancer Agents Med Chem. 2013;13:4-10.

95. Panel on Dietetic Products, Nutrition and Allergies (NDA). Scientific opinion on the tolerable upper intake level of vitamin D. EFSA J. 2012;10:1-45.

96. LeBlanc ES, Zakher B, Daeges M, Pappas M, Chou R. Screening for vitamin $D$ deficiency: a systematic review for the U.S. Preventive Services Task Force. Ann Intern Med. 2015;162:109-22. 